\title{
GDP job satisfaction
}

\author{
An assessment of career satisfaction among a group of general dental practitioners in Staffordshire \\ J. Gilmour, D. A. Stewardson, D. A. Shugars, F. J. T. Burke Br Dent J 2005; 198: 701-704
}

\section{Objectives}

To assess the level of job satisfaction among general dental practitioners from one area of England, and to assess the association of various personal and work related factors with job satisfaction.

Design

Postal questionnaire survey

Setting

General dental practices in South Staffordshire, Wolverhampton and Dudley, England.

Method

An anonymous questionnaire posted to all 396 registered dentists in the above areas.

\section{Results}

A 75\% response rate was achieved. Data were analysed using non-parametric statistics for any significant differences in the scores for stress, respect, overall professional satisfaction, quality of life and overall job satisfaction according to the different demographic groupings of the dentists (alpha $=0.05$ ). Dentists with an area of special interest had higher scores in all categories except quality of life. Overall job satisfaction was higher among private dentists, and those in group practices and in non-rural locations. The highest bi-variate correlation occurred between overall job satisfaction and overall professional satisfaction, delivery of care, income, respect and professional time.

\section{Conclusions}

Job satisfaction was judged to be good among this group. Stress was the factor associated with the greatest dissatisfaction. This survey produced similar results to preceding US studies, and suggests ways of improving job satisfaction.

\section{IN BRIEF}

- Overall job satisfaction among this group of dentists was good.

- Although stress is a feature of dentistry, many factors contribute to job satisfaction.

- Job satisfaction may be improved by developing an area of special interest by further training.

- Increasing the amount of treatment provided privately, and relocating to non-rural locations, may also improve job satisfaction. This would have obvious implications for the future of dentistry in the NHS, and for patients living in rural areas.

\section{COMMENT}

The structure of general dental practice is changing as Options for change ${ }^{1}$ and Delivering the changes ${ }^{2}$ are gradually implemented. In this new era of NHS dental service the focus will be on standards, services, prevention, accessibility and specialist care. A revolution is occurring in which practice will become patient-centred. In such climates of change little has been done to discover how it will affect GDPs' perceptions of satisfaction with their career choice. The importance of doing such work is not only for its intrinsic value but also to act as baseline with regard to job satisfaction and to aid planners to provide accessible, high quality dental care for all.

The work conducted by Gilmour and colleagues has done just that by supplying data on general dental practitioners (GDPs) working in Staffordshire. This survey provides a snapshot of how GDPs perceived their work situation. In general, those GDPs who had greater job satisfaction had greater professional satisfaction, income, respect from others and time for their patients and staff. Perhaps not unexpectedly, associates and assistants had greater quality of life scores compared with principals and GDPs whose practices were located in urban areas, who worked in group practices and who had private rather then NHS practices. The authors comment on the generalisability of their results and this would suggest that their findings have implications for all those working in general practice.

These findings are, therefore, of importance for those planning dental services and continuing professional development. Dental service planners must appreciate the role of isolation and boredom as factors in GDPs' professional satisfaction. ${ }^{3}$ These factors must be addressed if GDPs are to provide high quality accessible dental health services for their patient populations. There is a need to appreciate the intellectual as well as professional needs of GDPs. ${ }^{3}$ Gilmour etal. have demonstrated that GDPs who are involved in specialist practice had some of the highest levels of professional satisfaction. For those planning continuing professional development the message is: clear pathways must be made available for practitioners to take advantage in order to develop their clinical expertise and raise standards of patient care. If the revolution in dentistry is to be achieved then dental service planners and those planning dental education must take steps to ensure that dentists remain satisfied with their career choice Gilmour and colleagues have provided them with the first one.

R. Freeman, Professor of Dental Public Health, Queen's University Belfast

1. Department of Health. NHS dentistry: Options for change. London: DH, 2002.

2. Bedi R. NHS dentistry: Delivering the changes. London: DH, 2004

3. Burke FJ T, Freeman R. Preparing for dental practice. Oxford: Oxford University Press 2004.

doi: $10.1038 /$ sj.bdj.4812388 